\title{
Re: A Statewide Intervention to Reduce Hospitalizations after Prostate Biopsy
}

\author{
Paul R. Womble1, Susan M. Linsell1, Yuqing Gao1, Zaojun Ye1, James E. Montie1, \\ Tejal N. Gandhi2, Brian R. Lane3, Frank N. Burks4, David C. Miller5 \\ 1 University of Michigan, Department of Urology, Ann Arbor, Michigan \\ 2University of Michigan, Division of Infectious Disease, Ann Arbor, Michigan \\ 3Spectrum Health Medical Group, Department of Urology, Grand Rapids, Michigan \\ 4Oakland University William Beaumont School of Medicine, Department of Urology, Royal Oak, Michigan \\ 5 University of Michigan, Department of Urology, Ann Arbor, Michigan \\ Journal of Urology 2015;194:403-409
}

\section{EDITORIAL COMMENT}

Increasing number of patients having trans-rectal ultrasound biopsy (TRUS-BX) related serious infections. The main reason behind these infections may be the fluoroquinolone- resistant (FQR) bacteria. Incidence of FQR bacteria in rectal culture in Western and developing countries were 12\% and 40\%, respectively. The present study is first multi-centric prospective trial with a large cohort. Thirty centers were participated in the study. Group 1 ( $n=4087$ ) patients were used rectal culture targeted antibiotics and/or fluoroquinolone + additional antibiotics. Group $2(\mathrm{n}=5028)$ patients were used standard antibiotics prophylaxis. Two groups were compared for infection related hospitalization (IRH) within 30 days after TRUS-Bx.

The proportion of patients with IRH after TRUS-Bx decreased by 53\% in group 1 (0.56\% group 1 vs 1.19\% group 2, p=0.002). Group 1 was subdivided into targeted and augmented antibiotic prophylaxis groups. No statistically significant difference was found between targeted (0.47\%) and augmented (0.57\%) groups for IRH. Though in this study 53\% less hospitalization benefit was clearly shown for group 1. No advantage was confirmed in between targeted and augment prophylaxes for group 1. Emerging FQR should be kept in mind and prophylaxis alternatives should be considered.

\section{REFERENCES}

1. Urology, 85 (2015) 15-22. doi:10.1016/j.urology.2014.07.078

Tayyar Alp Özkan MD

\section{Re: Transperineal Template-Guided Prostate Biopsy: 10 Years of Experience}

\author{
Zhipeng Mai1, Weigang Yan1, Yi Zhou1, Zhien Zhou1, Jian Chen1, Yu Xiao2, Zhiyong Liang2, \\ Zhigang Ji1, Hanzhong Li1
}

1 Peking Union Medical College Hospital, Chinese Academy of Medical Sciences, Department of Urology, Beijing, China

2Peking Union Medical College Hospital, Chinese Academy of Medical Sciences, Department of Pathology, Beijing, China

BJU lnt 2014;18. doi: 10.1111/bju.13024. [Epub ahead of print]

\section{EDITORIAL COMMENT}

Transperineal prostate biopsy (TPB) is a sterile alternative to transrectal biopsy (TRUS-Bx). In addition, improved anterior-apical sampling and high cancer detection rate are area of interest for TPB. In the present study, the authors published 10 years of experience in 3007 TPB patients. Overall reported prostate cancer (PCa) detection rate was $35.5 \%$. The PCa detection rate in the apex (52\%) was higher than the average cancer detection rate in 10 defined regions ( $p=0.014)$. Anti-thrombotic treatment was not discontinued in the study population before TPB. Postoperative hematuria (47\%) and hematospermia (6.1\%) were the most frequent complications after biopsy. Perineal hematoma development was reported in $0.5 \%(n=16)$ of patients. Only one case of sepsis was reported in a long-term indwelling catheter patient. PCa was more common in transition zone. The present study demonstrated that transition zone cancer detection is statistical significantly higher with TPB. Particularly it is important for the re-biopsy patients. TPB is safe for patients with high risk of sepsis and for anti-thrombotic users. 